\title{
Sesterterpenes from the Sponge Dysidea sp.
}

\author{
Xiaoxia Yang ${ }^{\mathrm{a}}$, Zhiyu Shao ${ }^{\mathrm{a}, \mathrm{b}}$, and Xingqun Zhang ${ }^{\mathrm{a}, \mathrm{b}}$ \\ ${ }^{a}$ College of Chemistry, Chemical Engineering and Biotechnology, Donghua University, \\ Shanghai 201620, China \\ ${ }^{b}$ Key Laboratory of Science \& Technology of Eco-Textile, Ministry of Education, \\ Donghua University, Shanghai 201620, China
}

Reprint requests to Prof. Dr. X. Zhang. Fax: +86-21-67792608. E-mail: xqz@ dhu.edu.cn

Z. Naturforsch. 2010, 65b, 625-627; received December 23, 2009

\begin{abstract}
The acetone extract of the sponge Dysidea sp. was subjected to chromatography techniques for fractionation and purification. A new sesterterpene, scalarester (1), and four known scalaranes, viz. scalarin (2), scalaradial (3), desacetylscalaradial (4), and desacetoxyscalaradial (5), were obtained. Their structures have been elucidated by means of spectroscopic data interpretation, mainly 1D and 2D NMR and mass spectrometry.
\end{abstract}

Key words: Sesterterpene, Sponge, Dysidea sp., Scalarane

\section{Introduction}

Scalarane sesterterpenes are marine metabolites found either in sponges or in mollusks eaters of sponges. Many scalarane compounds play important ecological roles and also possess interesting biological properties, such as anti-inflammatory [1], antimicrobial [2], platelet aggregation inhibitory [3], and cytotoxic activities [4]. As part of our studies of biologically active metabolites of sponges, we now report the isolation and structural elucidation of one new sesterterpene, scalarester (1), and four known scalaranes (2-5) from Dysidea sp.

Dysidea sp. is a large genus widely distributed in tropical and subtropical waters around the world. We made a collection of the sponge Dysidea sp. from Sanya, Hainan Province, China.

\section{Results and Discussion}

Chromatography of the acetone fraction of the sponge Dysidea sp. resulted in the isolation of one novel sesterterpene (Fig. 1), scalarester (1), and four known sesterterpenes, scalarin (2), scalaradial (3), desacetylscalaradial (4), desacetoxyscalaradial (5).

Compound $\mathbf{1}$ was isolated as a colorless viscous oil. The molecular formula, $\mathrm{C}_{28} \mathrm{H}_{42} \mathrm{O}_{5}$, was established from HREIMS and ${ }^{13} \mathrm{C}$ NMR data. DEPT- ${ }^{13} \mathrm{C}$ NMR spectra (Table 1) showed twenty-eight carbon signals comprising seven methyls, seven methylenes, seven methines, and seven quaternary carbons, in which the

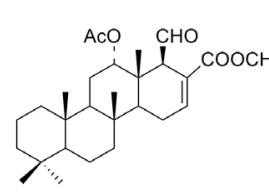

1

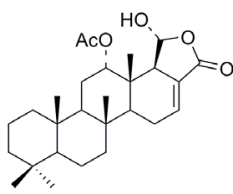

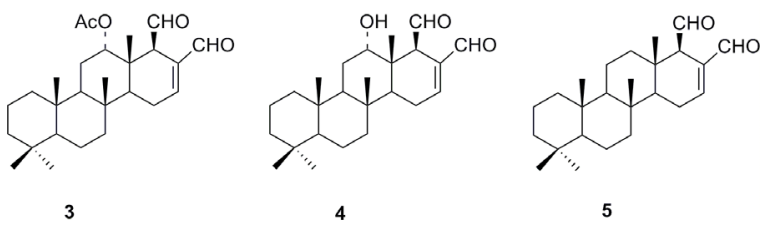

Fig. 1. Structures of scalarester (1), scalarin (2), scalaradial (3), desacetylscalaradial (4), and desacetoxyscalaradial (5).

functionalities of one aldehyde carbonyl $\left(\delta_{\mathrm{C}}=201.9\right.$, $\mathrm{C}-25)$, one acetoxy group $\left(\delta_{\mathrm{C}}=169.8, \mathrm{C}-26\right)$, one methyl ester carbonyl $\left(\delta_{\mathrm{C}}=167.8, \mathrm{C}-24\right)$ and one trisubstituted double bond $\left(\delta_{\mathrm{C}}=127.5,142.6\right)$ were distinguishable. The ${ }^{1} \mathrm{H}$ NMR spectrum showed one doublet signal for an aldehyde group at $\delta_{\mathrm{H}}=9.50(1 \mathrm{H}$, $\mathrm{d}, J=3.67 \mathrm{~Hz}$ ), one singlet for an olefinic hydrogen at $\delta_{\mathrm{H}}=7.15$, two broad signals for tertiary carbon hydrogen atoms at $\delta_{\mathrm{H}}=4.80$ and 3.59 , one singlet for a methyl ester groups at $\delta_{\mathrm{H}}=3.71$, one singlet for an acetoxyl group at $\delta_{\mathrm{H}}=2.16$, and five singlets for methyl groups at $\delta_{\mathrm{H}}=0.95,0.92,0.83,0.80$, and 0.79 . The signal at $\delta_{\mathrm{H}}=3.71(3 \mathrm{H}, \mathrm{s})$ correlated with the carbon at $\delta_{\mathrm{C}}=167.8$ in the HMQC spectrum and the carbon at $\delta_{\mathrm{C}}=127.5$ in the HMBC spectrum (Fig. 2), which indi- 
Table $1 .{ }^{13} \mathrm{C}$ NMR $(125 \mathrm{MHz})$ chemical shifts for $\mathbf{1}, \mathbf{2}$ and $\mathbf{3}$ in $\mathrm{CDCl}_{3}$.

\begin{tabular}{lrrrlrrr}
\hline & \multicolumn{1}{c}{$\mathbf{1}$} & \multicolumn{1}{c}{$\mathbf{2}$} & \multicolumn{1}{c}{$\mathbf{3}$} & & \multicolumn{1}{c}{$\mathbf{1}$} & \multicolumn{1}{c}{$\mathbf{2}$} & \multicolumn{1}{c}{$\mathbf{3}$} \\
\hline C-1 & 39.5 & 39.6 & 39.6 & C-2 & 17.8 & 17.9 & 17.9 \\
C-3 & 41.3 & 41.4 & 41.4 & C-4 & 33.2 & 33.2 & 33.2 \\
C-5 & 56.3 & 56.4 & 56.4 & C-6 & 18.2 & 18.4 & 18.3 \\
C-7 & 41.8 & 41.9 & 41.9 & C-8 & 36.6 & 37.7 & 36.7 \\
C-9 & 51.6 & 52.4 & 51.7 & C-10 & 37.7 & 37.2 & 37.8 \\
C-11 & 21.7 & 22.3 & 21.8 & C-12 & 74.8 & 74.5 & 74.8 \\
C-13 & 40.3 & 36.8 & 40.2 & C-14 & 49.0 & 49.7 & 49.4 \\
C-15 & 23.1 & 24.1 & 24.2 & C-16 & 142.6 & 135.3 & 153.0 \\
C-17 & 127.5 & 128.0 & 138.2 & C-18 & 53.3 & 50.8 & 52.1 \\
C-19 & 33.2 & 33.2 & 33.2 & C-20 & 21.3 & 21.4 & 21.3 \\
C-21 & 16.6 & 16.2 & 16.6 & C-22 & 16.2 & 16.0 & 16.2 \\
C-23 & 15.2 & 15.0 & 15.4 & C-24 & 167.8 & 167.8 & 193.0 \\
C-25 & 201.9 & 98.9 & 201.1 & C-26 & 169.8 & 171.2 & 169.8 \\
C-27 & 21.9 & 21.4 & 21.4 & C-28 & 52.3 & - & - \\
\hline
\end{tabular}

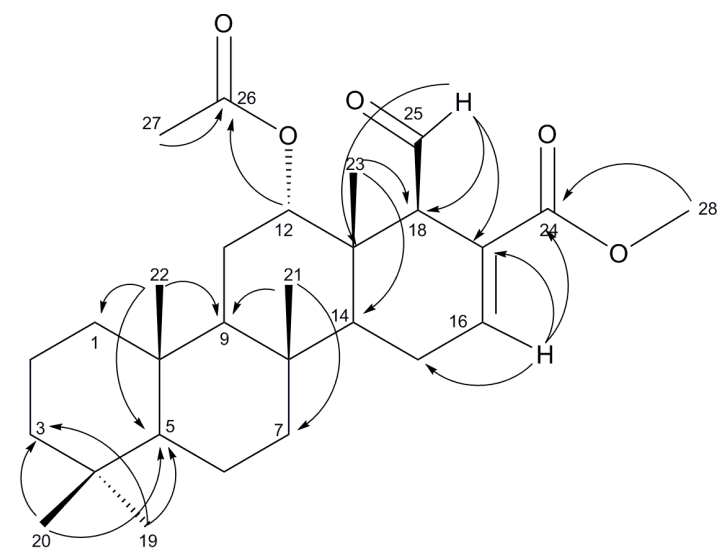

Fig. 2. The key HMBC correlations of scalarester (1).

cated the attachment of the methyl ester group at C-24. The signal at $\delta_{\mathrm{H}}=7.15(1 \mathrm{H}, \mathrm{m})$ was assigned to $\mathrm{H}-16$ from the HMBC correlation of $\mathrm{H}-16 / \mathrm{C}-17$, and the signal at $\delta_{\mathrm{H}}=2.16$ was assigned to $\mathrm{H}-27$ from the HMBC correlation of $\mathrm{H}-27 / \mathrm{C}-26$. The ${ }^{1} \mathrm{H}$ NMR and ${ }^{13} \mathrm{C}$ NMR spectra of compound 1 were similar to those of scalarin and scalaradial (Table 1). The ${ }^{1} \mathrm{H}$ NMR spectrum of $\mathbf{1}$ displayed a methyl ester groups at $\delta_{\mathrm{H}}=3.71(3 \mathrm{H}, \mathrm{s})$, and the ${ }^{13} \mathrm{C}$ NMR spectrum one aldehyde group at $\delta_{\mathrm{C}}=$ $201.9(\mathrm{C}-25)$ and a methyl ester carbonyl group at $\delta_{\mathrm{C}}=$ 167.8 (C-24) and 52.3 (C-28). A detailed analysis of the $2 \mathrm{D}$ NMR data indicated the structure deduced for 1 (scalarester) as shown in Fig 1.

Compounds 2-5 were isolated as colorless powders. Based on ${ }^{1} \mathrm{H}$ NMR, DEPT- ${ }^{13} \mathrm{C}$ NMR data and mass spectrometry the structures of the four compounds were established as scalarin, scalaradial, desacetylscalaradial and desacetoxyscalaradial, respectively. They have previously been isolated and char- acterized from the sponge Cacospongia scalaris. The structures of $\mathbf{2}-\mathbf{5}$ were confirmed by comparing their spectral data with those presented in references [5-8].

\section{Experimental Section}

General

NMR spectra were measured on a Bruker DRX-400 spectrometer with the residual $\mathrm{CDCl}_{3}$ signal $\left(\delta_{\mathrm{H}}=7.26 \mathrm{ppm}\right.$, $\left.\delta_{\mathrm{C}}=77.0 \mathrm{ppm}\right)$ as an internal standard. ESIMS spectra were recorded on a Q-TOF Micro LC-MS mass spectrometer. HREIMS spectra were performed on a Micromass Q-TOF spectrometer. Melting points were determined on a Fisher-Johns micromelting point apparatus and were uncorrected. Silica gel (200 - 300 mesh) was purchased from Qingdao Marine Chemical Co. (Qing-dao, China). All solvents used for extraction and isolation were of analytical grade, purchased from Sinopharm Chemical Reagent Co., Shanghai, China.

\section{Animal material}

Specimens of Dysidea sp. were collected from Sanya, Hainan Province, China, in South China Sea, in 2006, and kept frozen prior to extraction. The sponge material was identified as Dysidea sp. by Dr. Zaizhong Chen from Shanghai Ocean University. A voucher specimen (registry No Dsp03) was deposited at the Chemistry and Biological Engineering Department, Donghua University.

\section{Extraction and isolation}

The bodies of the sponge Dysidea sp. ( $85 \mathrm{~g}$, dry wt. after extraction) were chopped, then soaked in acetone $(800 \mathrm{~mL})$ and extracted at r.t. with sonication for $1 \mathrm{~h}$. The filtered acetone solution was evaporated under reduced pressure, and the residue was extracted four times with ether $(4 \times 100 \mathrm{~mL})$. The solvent was evaporated to give an oily residue (3.2 g), which was subjected to silica gel column chromatography using a petroleum ether-EtOAc gradient as eluent. The fraction was eluted with petroleum ether-EtOAc $(9: 1)$ to give compound $5(6.1 \mathrm{mg}, 0.007 \%$ of dry material $)$. The fraction eluted with petroleum ether-EtOAc $(8.5: 1.5)$ was further purified by preparative TLC (silica gel) to yield compound 3 (18 mg, $0.021 \%$ of dry material), compound 4 (12 mg, $0.014 \%$ of dry material) and compound $1(4.5 \mathrm{mg}, 0.005 \%$ of dry material). The fraction eluted with petroleum etherEtOAc (8:2) was re-chromatographed on a silica gel column eluting with petroleum ether-EtOAc (7.5:2.5) to give compound $2(5.1 \mathrm{mg}, 0.006 \%$ of dry material $)$.

\section{Scalarester (1)}

Colorless oil, $[\alpha]_{\mathrm{D}}=+44.2\left(c=0.01, \mathrm{CH}_{3} \mathrm{OH}\right) .-{ }^{1} \mathrm{H}$ $\operatorname{NMR}\left(\mathrm{CDCl}_{3}, 400 \mathrm{MHz}\right): \delta=9.50(1 \mathrm{H}, \mathrm{d}, J=3.67 \mathrm{~Hz}$, 
$\mathrm{H}-25), 7.15$ (1H, m, H-16), 4.80 (1H, m, H-12), $3.71(3 \mathrm{H}$, s, H-28), 3.59 (1H, m, H-18), 2.16 (3H, s, H-27), 0.95 $(3 \mathrm{H}, \mathrm{s}), 0.92(3 \mathrm{H}, \mathrm{s}), 0.83(3 \mathrm{H}, \mathrm{s}), 0.80(3 \mathrm{H}, \mathrm{s}), 0.79(3 \mathrm{H}$, s). $-{ }^{13} \mathrm{C} \mathrm{NMR}\left(\mathrm{CDCl}_{3}, 150 \mathrm{MHz}\right): \delta=201.9(\mathrm{C}-25)$, 169.8 (C-26), 167.8 (C-24), 142.6 (C-16), 127.5 (C-17), 74.8 (C-12), 56.3 (C-5), 52.3 (C-28), 51.6 (C-9), 49.0 (C-14), 41.8 (C-7), 41.3 (C-3), 40.3 (C-13), 39.5 (C-1), 37.7 (C-10), 36.6 (C-8), 33.2 (C-4), 33.2 (C-19), 23.1 (C-15), 21.9 (C-27), 21.7 (C-11), 21.3 (C-20), 18.2 (C-6), 17.8 (C-2), 16.6 (C-21), 16.2 (C-22), $15.2(\mathrm{C}-23) .-\mathrm{MS}((+)-\mathrm{ESI}): \mathrm{m} / \mathrm{z}=459[\mathrm{M}+\mathrm{H}]^{+}$, $481[\mathrm{M}+\mathrm{Na}]^{+} .-\mathrm{HRMS}((+)-\mathrm{EI}): \mathrm{m} / z=458.3015$ (calcd: 458.3032 for $\left.\mathrm{C}_{28} \mathrm{H}_{42} \mathrm{O}_{5},[\mathrm{M}]^{+}\right)$.

\section{Scalarin (2)}

Colorless powder, m. p. $133-135{ }^{\circ} \mathrm{C} .-{ }^{1} \mathrm{H} \mathrm{NMR}\left(\mathrm{CDCl}_{3}\right.$, $400 \mathrm{MHz}): \delta=6.82(1 \mathrm{H}, \mathrm{d}, J=2.89 \mathrm{~Hz}, \mathrm{H}-16), 5.69(1 \mathrm{H}$, br, H-25), 4.92 (1H, m, H-12), $3.12(1 \mathrm{H}, \mathrm{s}, \mathrm{H}-18), 2.08$ $(3 \mathrm{H}, \mathrm{s}, \mathrm{H}-27), 0.96(3 \mathrm{H}, \mathrm{s}), 0.85(3 \mathrm{H}, \mathrm{s}), 0.84(3 \mathrm{H}, \mathrm{s}), 0.81$ $(3 \mathrm{H}, \mathrm{s}), 0.79(3 \mathrm{H}, \mathrm{s}) .-{ }^{13} \mathrm{C} \mathrm{NMR}\left(\mathrm{CDCl}_{3}, 150 \mathrm{MHz}\right): \delta=$ 171.2 (C-26), 167.8 (C-24), 135.3 (C-16), 128.0 (C-17), 98.9 (C-25), 74.5 (C-12), 56.4 (C-5), 52.4 (C-9), 50.8 (C-18), 49.7 (C-14), 41.9 (C-7), 41.4 (C-3), 39.6 (C-1), 37.7 (C-8), 37.2 (C-10), 36.8 (C-13), 33.2 (C-4), 33.2 (C-19), 24.1 (C-15), 22.3 (C-11), 21.4 (C-20), 21.4 (C-27), 18.4 (C-6), 17.9 (C-2), 16.2 (C-21), $16.0(\mathrm{C}-22), 15.0(\mathrm{C}-23) .-\mathrm{MS}((+)-\mathrm{ESI}): \mathrm{m} / \mathrm{z}=$ $445[\mathrm{M}+\mathrm{H}]^{+}, 467[\mathrm{M}+\mathrm{Na}]^{+}$.

\section{Scalaradial (3)}

Colorless needless, m.p. $110-112{ }^{\circ} \mathrm{C} . \quad-{ }^{1} \mathrm{H}$ NMR $\left(\mathrm{CDCl}_{3}, 400 \mathrm{MHz}\right): \delta=9.51(1 \mathrm{H}, \mathrm{d}, J=3.9 \mathrm{~Hz}, \mathrm{H}-25), 9.45$ $(1 \mathrm{H}, \mathrm{s}, \mathrm{H}-24), 7.05(1 \mathrm{H}, \mathrm{dd}, J=5.4,2.2 \mathrm{~Hz}, \mathrm{H}-16), 4.75(1 \mathrm{H}$, $\mathrm{d}, J=1.9 \mathrm{~Hz}, \mathrm{H}-12), 3.50$ (1H, s, H-18), 2.14 (3 H, s, H-27), $0.96(3 \mathrm{H}, \mathrm{s}), 0.95(3 \mathrm{H}, \mathrm{s}), 0.83(3 \mathrm{H}, \mathrm{s}), 0.79(3 \mathrm{H}, \mathrm{s}), 0.77$ $(3 \mathrm{H}, \mathrm{s}) .-{ }^{13} \mathrm{C} \mathrm{NMR}\left(\mathrm{CDCl}_{3}, 150 \mathrm{MHz}\right): \delta=201.1(\mathrm{C}-25)$, 193.1 (C-24), 169.8 (C-26), 153.0 (C-16), 138.2 (C-17), 74.8 (C-12), 56.5 (C-5), 52.1 (C-18), 51.8 (C-9), 49.4 (C-14), 41.9
(C-7), 41.4 (C-3), 40.2 (C-13), 39.7 (C-1), 37.8 (C-10), 36.7 (C-8), 33.2 (C-4), 33.2 (C-19), 24.2 (C-15), 21.9 (C-11), 21.5 (C-27), 21.3 (C-20), 18.4 (C-6), 17.9 (C-2), 16.7 (C-21), 16.2 (C-22), $15.4(\mathrm{C}-23) .-\mathrm{MS}((+)-\mathrm{ESI}): m / z=429[\mathrm{M}+\mathrm{H}]^{+}, 451$ $[\mathrm{M}+\mathrm{Na}]^{+}$.

\section{Desacetylscalaradial (4)}

Colorless powder, m.p. $198-200{ }^{\circ} \mathrm{C} .-{ }^{1} \mathrm{H} \mathrm{NMR}\left(\mathrm{CDCl}_{3}\right.$, $400 \mathrm{MHz}): \delta=9.54(1 \mathrm{H}, \mathrm{d}, J=4.8 \mathrm{~Hz}, \mathrm{H}-25), 9.47(1 \mathrm{H}$, s, H-24), $7.05(1 \mathrm{H}, \mathrm{dd}, J=5.4,2.2 \mathrm{~Hz}, \mathrm{H}-16), 3.60(1 \mathrm{H}$, brs, H-12), $3.46(1 \mathrm{H}, \mathrm{s}, \mathrm{H}-18), 0.92(3 \mathrm{H}, \mathrm{s}), 0.85(3 \mathrm{H}$, s), $0.82(3 \mathrm{H}, \mathrm{s}), 0.80(3 \mathrm{H}, \mathrm{s}), 0.77(3 \mathrm{H}, \mathrm{s}) .-{ }^{13} \mathrm{C} \mathrm{NMR}$ $\left(\mathrm{CDCl}_{3}, 150 \mathrm{MHz}\right): \delta=204.9(\mathrm{C}-25), 193.3(\mathrm{C}-24), 153.9$ (C-16), 138.1 (C-17), 71.7 (C-12), 56.2 (C-5), 52.7 (C-18), 50.7 (C-9), 48.7 (C-14), 41.9 (C-7), 41.4 (C-3), 40.2 (C-13), 39.5 (C-1), 37.7 (C-10), 36.7 (C-8), 33.2 (C-4), 33.2 (C-19), 24.6 (C-15), 24.21 (C-11), 21.3 (C-20), 18.4 (C-6), 18.0 (C-2), 16.3 (C-21), 16.2 (C-22), 15.4 (C-23). - MS ((+)-ESI): $\mathrm{m} / \mathrm{z}=387[\mathrm{M}+\mathrm{H}]^{+}, 409[\mathrm{M}+\mathrm{Na}]^{+}$.

\section{Desacetoxyscalaradial (5)}

Colorless powder, m.p. $201-203{ }^{\circ} \mathrm{C} .-{ }^{1} \mathrm{H} \mathrm{NMR}\left(\mathrm{CDCl}_{3}\right.$, $400 \mathrm{MHz}): \delta=9.53(1 \mathrm{H}, \mathrm{d}, J=4.6 \mathrm{~Hz}, \mathrm{H}-25), 9.46(1 \mathrm{H}$, s, H-24), $7.04(1 \mathrm{H}, \mathrm{dd}, J=5.4,2.2 \mathrm{~Hz}, \mathrm{H}-16), 2.80(1 \mathrm{H}$, brs, H-12), $0.95(3 \mathrm{H}, \mathrm{s}), 0.91(3 \mathrm{H}, \mathrm{s}), 0.84(3 \mathrm{H}, \mathrm{s}), 0.83$ $(3 \mathrm{H}, \mathrm{s}), 0.80(3 \mathrm{H}, \mathrm{s}) .-{ }^{13} \mathrm{C} \mathrm{NMR}\left(\mathrm{CDCl}_{3}, 100 \mathrm{MHz}\right): \delta=$ 202.0 (C-25), 193.1 (C-24), 154.4 (C-16), 138.0 (C-17), 60.9 (C-9), 60.7 (C-18), 56.4 (C-5), 54.2 (C-14), 42.0 (C-3), 41.6 (C-7), 41.1 (C-12), 39.8 (C-1), 37.8 (C-13), 37.4 (C-10), 36.9 (C-8), 33.2 (C-4), 33.2 (C-19), 24.3 (C-15), 21.3 (C-20), 18.5 (C-2), 18.0 (C-6), 17.0 (C-23), 17.1 (C-11), 16.4 (C-22), 16.1 (C-21). $-\mathrm{MS}((+)-\mathrm{ESI}): \mathrm{m} / \mathrm{z}=371[\mathrm{M}+\mathrm{H}]^{+}, 393[\mathrm{M}+\mathrm{Na}]^{+}$.

\section{Acknowledgement}

This work was supported by Key Laboratory of Science \& Technology of the Eco-Textile Foundation, Ministry of Education, Donghua University.
[1] A. Fontana, E. Mollo, J. Ortea, M. Gavagnin, G. Cimino, J. Nat. Prod. 2000, 63, 527-530.

[2] S. Wonganuchitmeta, S. Yuenyongsawad, N. Keawpradub, A. Plubrukarn, J. Nat. Prod. 2004, 67, $1767-$ 1770.

[3] M. Nakagawa, Y. Hamamoto, M. Ishihama, S. Hamasaki, M. Endo, Tetrahed. Lett. 1987, 28, $431-434$.

[4] J. Song, W. Jeong, N. Wang, H.-S. Lee, C. J. Sim, K.-B. Oh, J. Shin, J. Nat. Prod. 2008, 71, 1866-1871.
[5] E. Fattorusso, C. Magno, C. Santacroce, D. Sica, Tetrahedron 1972, 28, $5993-5997$.

[6] G. Cimino, S. De Stefano, L. Minale, Experientia 1974, 30, 846-847.

[7] F. Yasuda, H. Tada, Experientia 1981, 37, 110-111.

[8] S. De Rosa, R. Puliti, A. Crispino, A. De Giulio, C. A. Mattia, L. Mazzarella, J. Nat. Prod. 1994, 57, 256262. 